\title{
Preliminary Studies on Liquiritin, Deoxyschizandrin, and Tanshinone II A as Potential Anti-Neurodegenerative Disease Agent: Determination by Reverse-Phase Liquid Chromatography in Tianwang Buxin Pills
}

\author{
Dan Xu $\mathbb{D}^{1},{ }^{1}$ Sicen Wang, ${ }^{2}$ Xiaofang Hou $\mathbb{D}^{2},{ }^{2}$ and Changshan Sun $\mathbb{D}^{3}$ \\ ${ }^{1}$ School of Traditional Chinese Medicine, Shenyang Pharmaceutical University, Shenyang 110016, China \\ ${ }^{2}$ School of Pharmacy, Xi'an Jiaotong University, Xi'an 710061, China \\ ${ }^{3}$ School of Pharmacy, Shenyang Pharmaceutical University, Shenyang 110016, China
}

Correspondence should be addressed to Xiaofang Hou; houxiaofang@xjtu.edu.cn and Changshan Sun; sunchangshan_spu@126.com

Received 13 February 2019; Revised 16 July 2019; Accepted 22 July 2019; Published 5 August 2019

Academic Editor: Antony C. Calokerinos

Copyright (C) 2019 Dan Xu et al. This is an open access article distributed under the Creative Commons Attribution License, which permits unrestricted use, distribution, and reproduction in any medium, provided the original work is properly cited.

\begin{abstract}
Tianwang Buxin pill (TWBXP) is an ancient Chinese classic prescription. Liquiritin, deoxyschizandrin, and tanshinone II A are three bioactive components in TWBXP, which have been proven to be closely related to the therapy effect of neurodegenerative disease. Their contents are very low in TWBXP. In this study, we used a diode array detector (DAD) to perform a full wavelength scanning in order to choose a most suitable detection wavelength to establish an HPLC method for the simultaneous determination of these three components in TWBXP. Various chromatographic conditions were investigated to verify its applicability. Finally, a Kromasil C18 column $(250 \times 4.6 \mathrm{~mm}, 5 \mu \mathrm{m})$ thermostated at $30^{\circ} \mathrm{C}$, mobile phase as $0.2 \%$ phosphoric acid solution (eluent A), and $0.1 \%$ phosphoric acid-acetonitrile solution (eluent B) were used. Both external standard method and internal standard method were used for quantification. The results showed that both methods were simple and convenient in operation without special pretreatment and exhibits excellent precision, repeatability (RSD $<3.0 \%)$, good linearity $\left(R^{2}>0.9990\right)$, and good recoveries (recovery value between 95\% and 105\%). Because of the low contents in samples, the internal standard method provided a better accurate result than the external standard method. The stability results showed the sample became stable within 24 hours at room temperature. The method provides a convenient and effective way for the quality control of TWBXP, and it can help the research about $\mathrm{AD}$ in the future.
\end{abstract}

\section{Introduction}

Tianwang Buxin pill (TWBXP) is an ancient Chinese classic prescription, which was recorded in She Sheng Mi Pou of Ming Dynasty. The prescription has been passed down to the present day with exquisite and excellent curative effect on treating heart-yin deficiency, heart palpitations, forgetfulness, insomnia, much dream, dry stool, etc [1]. With the increasing attention and research on TWBXP, many researchers have discovered that TWBXP possessed various significant effects such as antioxidant [2], antiaging [3, 4], antidepressant [5], and cardiovascular protecting effects [6-8]. Therefore, TWBXP has a huge market in Chinese.
According to the record, it is composed of 16 traditional Chinese medicines (TCMs).

Three bioactive components in TWBXP, liquiritin, deoxyschizandrin, and tanshinone II A, have been proven to be closely related to the therapy effect of neurodegenerative disease. Liquiritin is a major ingredient in Glycyrrhizae radix which has been proven to significantly ameliorate spatial learning, cognitive ability, and memory impairment of rats with Alzheimer's disease [9]. It possessed various pharmacological activities and exhibited various positive biological effects, including neuroprotective [10], anticancer [11], and antidepressant [12] effect. Deoxyschizandrin is a lignans ingredient in Schisandrae Chinensis Fructus, with antiaging 
effect [13], hepatoprotective activity [14] and anticancer effect [15], and it has been experimentally proven that it can significantly improve $A \beta_{1-42}$-induced short-term and spatial memory impairments in the Y-maze and water maze tests [16]. Tanshinone II A is a major ingredient in Salviae Miltiorrhizae Radix et Rhizoma which has strong antioxidative activity [17] and neuroprotective effect [18-20]. It can improve the cognitive dysfunction in learning and memory exhibited and ameliorating spatial memory impairment by the $\mathrm{AD}$ rats. The possible mechanism is tanshinone II A could ameliorate the synaptic deficit at the early phase of $\mathrm{AD}$ and attenuates $\mathrm{AD}$-related protein expression [21-25].

Due to the pharmacological effects of liquiritin, deoxyschizandrin, and tanshinone II A, the simultaneous determination of three bioactive components in TWBXP is significant to disclose the secret underlying its efficacy on neurodegenerative disease. Sun et al. determined 6 lignans (schisandrin, schisandrol B, schisantherin A, deoxyschisandrin, schisandrin B, and schisandrin C) in TWBXP [26] by RPLC. Yan et al. determined four components (tanshinone II A, cryptotanshinone, salvianolic acid B, and tanshinone I) of TWBXP by UPLC-Q-TOF-MS [27]. So far, as we know, no one has reported about simultaneous determination of the components related to $\mathrm{AD}$ treatment.

In this study, we established an HPLC method for the simultaneous determination of these three components in TWBXP. Various chromatographic conditions were investigated to verify its applicability. According to the previous experiments, the three active component contents were very low. Quantification of these three components was verified with both the internal standard method and external standard method. The method provides a convenient and effective way for the quality control of TWBXP, and it can help the research about $\mathrm{AD}$ in the future.

\section{Experiments}

2.1. Instruments. 1100 model Agilent HPLC was equipped with LC-G13 solvent delivery unit, G1313A autosampler, G1315B diode array detector, and LC chromatography workstation. Kromasil C18 column $(250 \times 4.6 \mathrm{~mm}, 5 \mu \mathrm{m})$ and Agilent C18 column $(4.6 \times 250 \mathrm{~mm}, 5 \mu \mathrm{m})$ were used. BT25S model electronic scale was from Sartorius. KQ5200E model ultrasonic cleaner was from Kunshan Ultrasonic Instrument Co., Ltd. HH-2 model digital circulating water bath was from Jintan Ronghua Instrument Manufacturing Co., Ltd. RE52CS model rotary evaporator was purchased from Shanghai Yarong Biochemical Instrument Factory. ZDHW model heating mantle and 101 model electric blastdrying oven were from Beijing Zhongxing Weiye Instrument Co., Ltd. High speed crusher was from Tianjin Taisite Instrument Co., Ltd.

2.2. Materials. HPLC-grade acetonitrile was supplied by Sigma-Aldrich (USA). Analytical grade ethanol was supplied by the Yuwang Group (Shandong). Phosphoric acid was Tianjin Hengxing Chemical Preparation Co. Ltd. Liquiritin (purity $>98 \%$ ), deoxyschizandrin (purity $>98 \%$ ), tanshinone II A (purity $>98 \%$ ), and berberine (purity $>98 \%$ ) reference substances were purchased from China National Institute for the Control of Pharmaceutical and Biological Products. Tianwang Buxin pills were purchased from Beijing Tongrentang Group.

2.3. Sample Treatment. The stock solutions of liquiritin $(500 \mu \mathrm{g} / \mathrm{mL})$, deoxyschizandrin $(300 \mu \mathrm{g} / \mathrm{mL})$, tanshinone II A $(500 \mu \mathrm{g} / \mathrm{mL})$, and the internal standard (IS) berberine $(1500 \mu \mathrm{g} / \mathrm{mL})$ were prepared by dissolving suitable quantities of the standard substance in methanol. The series mixed standard working solutions for the standard curve were prepared by diluting the stock solution of liquiritin, deoxyschizandrin, and tanshinone II A at six concentration levels corresponding to $10,20,50,75,100$, and $150 \mu \mathrm{g} / \mathrm{mL}$ of liquiritin; $15,30,75,112.5,150$, and $180 \mu \mathrm{g} / \mathrm{mL}$ of deoxyschizandrin; and $5,25,55,90,125$, and $150 \mu \mathrm{g} / \mathrm{mL}$ of tanshinone II A. IS was spiked into the mixed standard working solutions at $750 \mu \mathrm{g} / \mathrm{mL}$. All the standard working solutions were labeled as $1,2,3,4,5$, and 6 and stored at $4^{\circ} \mathrm{C}$ before use.

TWBXP was cut into little pieces, accurately weighted (about $9 \mathrm{~g}$ ) and transferred into the $100 \mathrm{~mL}$ round-bottom flask. They were extracted with $50 \mathrm{~mL}$ methanol in an ultrasonic bath for $30 \mathrm{~min}$ and diluted to $50 \mathrm{~mL}$ by methanol. All samples were filtered through a $0.22 \mu \mathrm{m}$ microporous membrane syringe filter (Shenyang Chromatography Scientific Instrument Co., Ltd.) and spiked with $750 \mu \mathrm{g} / \mathrm{mL}$ berberine before injecting into the HPLC system for analysis.

\subsection{Establishment of an HPLC Method for the Determination} of Three Active Components. The analyses were carried out on an 1100 model Agilent HPLC equipped with the LC-G13 solvent delivery unit, G1313A autosampler, G1315B diode array detector, and LC chromatography workstation. After optimization of detection wavelength, column type, and elution gradient (Table 1), the chromatographic separation was performed using a Kromasil C18 column $(250 \times 4.6 \mathrm{~mm}$, $5 \mu \mathrm{m}$ ) thermostated at $30^{\circ} \mathrm{C}$, mobile phase as $0.2 \%$ phosphoric acid solution (eluent $\mathrm{A}$ ), and $0.1 \%$ phosphoric acidacetonitrile solution (eluent $\mathrm{B}$ ). The gradient elution program was from $0 \%$ to $15 \%$ B in $10 \mathrm{~min}$, was from $0 \%$ to $30 \%$ $\mathrm{B}$ at $30 \mathrm{~min}$, was from $0 \%$ to $75 \% \mathrm{~B}$ at $70 \mathrm{~min}$, remained at $75 \% \mathrm{~B}$ to $85 \mathrm{~min}$, returned to $0 \% \mathrm{~B}$ at $86 \mathrm{~min}$, and was in column equilibration for $14 \mathrm{~min}$. The wavelength was set at $203 \mathrm{~nm}$. The injection volume was $5 \mu \mathrm{L}$, and the flow rate was set at $1 \mathrm{~mL} / \mathrm{min}$.

\subsection{Validation of the HPLC Method}

2.5.1. External Standard Method. To validate the HPLC method for determination of liquiritin, deoxyschizandrin, and tanshinone II A in TWBXP, the specificity, linearity, precision, accuracy, and repeatability of the method were studied. A volume of $5 \mu \mathrm{L}$ of each mixed standard working solution $(1,2,3,4,5$, and 6$)$ was injected under the operating chromatographic conditions previously described. The 
TABLE 1: Optimization of gradient conditions.

\begin{tabular}{|c|c|c|c|}
\hline Chromatographic factor & Conditions & Optimized condition & Reason \\
\hline Elution gradient & $\begin{array}{c}\text { (1) } 0-10 \mathrm{~min}, 0-15 \% \mathrm{~B} ; 10-30 \mathrm{~min}, 15-30 \% \mathrm{~B} \text {; } \\
30-70 \mathrm{~min}, 30-75 \% \text {; } 70-85 \mathrm{~min}, 75 \% \mathrm{~B} ; 85-86 \mathrm{~min} \text {, } \\
75-0 \% \mathrm{~B} ; 86-100 \mathrm{~min}, 0 \% \mathrm{~B} \text {; } \\
\text { (2) } 0-10 \mathrm{~min}, 0-15 \% \mathrm{~B} ; 10-30 \mathrm{~min}, 15-30 \% \mathrm{~B} \text {; } \\
30-60 \mathrm{~min}, 30-65 \% \text {; } 60-70 \mathrm{~min}, 65-70 \% \mathrm{~B} \text {; } \\
70-85 \mathrm{~min}, 70-75 \% \mathrm{~B} ; 85-86 \mathrm{~min}, 75-0 \% \mathrm{~B} \\
86-100 \mathrm{~min}, 0 \% \mathrm{~B}\end{array}$ & Procedure (1) & High peak resolution \\
\hline
\end{tabular}

calibration curve was established by plotting the peak area versus the corresponding concentration.

2.5.2. Internal Standard Method. $5 \mu \mathrm{L}$ of each series mixed standard working solution $(1,2,3,4,5$, and 6$)$ spiked with $750 \mu \mathrm{g} / \mathrm{mL}$ berberine was injected under the operating chromatographic conditions previously described. Linearity of each calibration curve was determined by plotting the peak area ratio of analytes to IS versus the concentration of analytes to IS with weighted linear regression.

Certain acceptance limits were developed for linearity, precision, repeatability, and accuracy. The LODs and LOQs of liquiritin, deoxyschizandrin, and tanshinone II A were estimated based on the signal-to-noise ratio criterion $\mathrm{S} / \mathrm{N}=3$ and 10, respectively. The correlation coefficient for linearity, limits of precision and repeatability, and accuracy value has all been calculated.

The percent recovery value was calculated using the equation below:

$$
\text { recovery }=\frac{(\text { found amount }- \text { initial amount })}{\text { added amount }} \cdot 100 \%
$$

2.6. Stability Study of Tianwang Buxin Pills Preparations. The stability of TWBXP solution stored at room temperature was studied at $0,2,4,8,12$, and $24 \mathrm{~h}$, respectively. The stability limit was set to be $<3.0 \%$ at RSD (\%) in 24 hours at $25^{\circ} \mathrm{C}$ and $40 \% \mathrm{RH}$.

2.7. Determination of the Real Samples. Eight batches of the TWBXP sample were prepared according to 2.3. The sample solutions $(5 \mu \mathrm{L})$ spiked with $750 \mu \mathrm{g} / \mathrm{mL}$ berberine were injected into the HPLC system; the contents of liquiritin, deoxyschizandrin, and tanshinone II A in TWBXP were determined by both external standard and internal standard methods.

\section{Results and Discussion}

3.1. Optimization of the Chromatographic Separation. Columns, UV wavelength, and elution gradient were studied in our work (Table 1). Considering the absorption characteristics of liquiritin, deoxyschizandrin, and tanshinone II A, $203 \mathrm{~nm}$ was chosen as the detection wavelength. Optimization of the column type and elution gradient results is shown in Table 1. Using the optimized conditions, linearity, precision, repeatability, and accuracy were tested. The representative chromatograms of the mixed standard are illustrated in Figure 1. All peaks were baseline separated and had a good resolution $(R \geq 1.5)$ under the chromatographic conditions. The retention time of standard liquiritin, deoxyschizandrin, and tanshinone II A was $22.8 \mathrm{~min}$, $68.2 \mathrm{~min}$, and $71.7 \mathrm{~min}$, respectively.

3.2. Method Validation. The linearity of the method was evaluated at $\lambda=203 \mathrm{~nm}$ using the mixed standard solutions. The results including the regression equations, the linear ranges, and regression coefficients are summarized in $\mathrm{Ta}-$ bles 2 and 3 .

For linearity, the correlation coefficient $\left(R^{2}\right)$ was above 0.99 by both the external standard method and internal standard method. The precision (Table 4) and repeatability (Table 5) were tested. In the precision experiment, the RSD value of liquiritin, deoxyschizandrin, and tanshinone II A was $1.23 \%, 1.88 \%$, and $1.43 \%$, respectively $(n=6)$. When it comes to repeatability, the mean concentration of liquiritin, deoxyschizandrin, and tanshinone II A in TWBXP was $0.141 \mathrm{mg} / \mathrm{g}, 0.129 \mathrm{mg} / \mathrm{g}$, and $0.308 \mathrm{mg} / \mathrm{g}$, while the RSD of which was $1.76 \%, 2.00 \%$, and $1.95 \%$, respectively.

Recovery studies of liquiritin, deoxyschizandrin, and tanshinone II A were evaluated by analysis of samples of each analyte. The data were calculated with equation (1) and are summarized in Table 6. The recovery range of liquiritin, deoxyschizandrin, and tanshinone II A was 99.22\% $102.92 \%$, $100.48 \% \sim 105.28 \%$, and $96.27 \% \sim 100.03 \%$, respectively. The RSD value was $1.50 \%$.

3.3. Stability Study. The stability test result is shown in Table 7; the RSD value of liquiritin, deoxyschizandrin, and tanshinone II A was $1.18 \%, 1.34 \%$, and $1.88 \%$, respectively. The results indicate the sample becomes stable within 24 hours at $25^{\circ} \mathrm{C}$ and $40 \% \mathrm{RH}$.

3.4. Sample Analysis and Comparison of Two Methods. The chromatograms of eight batches samples are shown in Figure 2. The peaks of liquiritin, deoxyschizandrin, and tanshinone II A are labeled as 1, 2, and 3. Table 8 shows the contents of these three active components: liquiritin, deoxyschizandrin, and tanshinone II A under the external standard method and internal standard method, respectively.

Normally, the internal standard method is used in biological samples or some other matrix samples. In this case, we can see that the contents of these three active components 


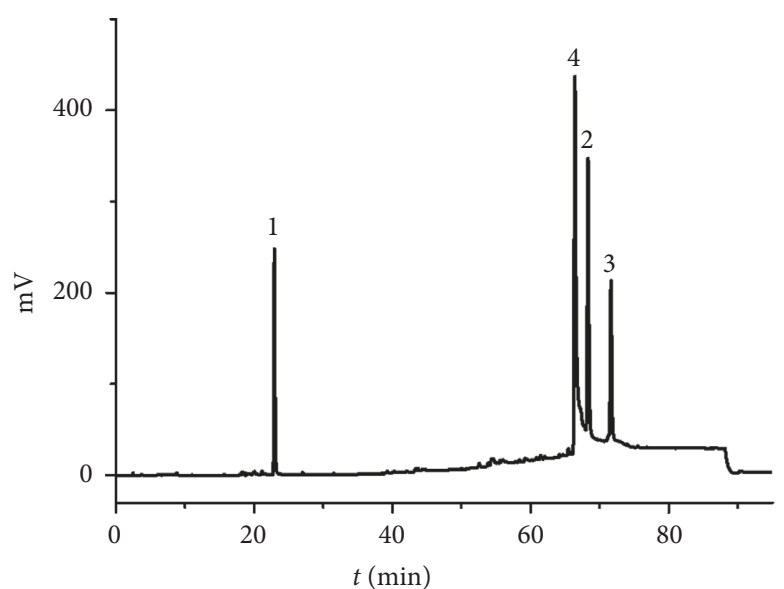

FIgURE 1: HPLC chromatogram of the mixed standard solution of liquiritin (1), deoxyschizandrin (2), tanshinone II A (3), and internal standard berberine (4).

TABle 2: The Calibration curve of liquiritin, deoxyschizandrin, and tanshinone II A under the external standard method.

\begin{tabular}{lcccccc}
\hline & Range $(\mu \mathrm{g} / \mathrm{mL})$ & Slope $(\text { mean } \pm \mathrm{SD})^{\mathrm{b}}$ & ${\text { Intercept }(\mathrm{mean} \pm \mathrm{SD})^{\mathrm{b}}}$ & $R^{2}$ & $\mathrm{LOD}^{\mathrm{a}}\left(\mu \mathrm{g} / \mathrm{mL}^{2}\right.$ & $\mathrm{LOQ}{ }^{\mathrm{a}}(\mu \mathrm{g} / \mathrm{mL})$ \\
\hline Liquiritin & $10.00-150.00$ & $7.20 \pm 0.41$ & $23.14 \pm 1.52$ & 0.9994 & 3 & 10 \\
Deoxyschizandrin & $15.00-180.00$ & $9.45 \pm 0.11$ & $21.77 \pm 0.62$ & 0.9984 & 5 & 15 \\
Tanshinone II A & $5.00-150.00$ & $6.07 \pm 0.06$ & $30.90 \pm 1.05$ & 0.999 & 1.5 & 4.5 \\
\hline
\end{tabular}

${ }^{\mathrm{a}}$ Limit of detection (LOD) at $\mathrm{S} / \mathrm{N}=3$ and limit of quantification (LOQ) at $\mathrm{S} / \mathrm{N}=10$. ${ }^{\mathrm{b}}$ Data are presented as mean $\pm \mathrm{SD}(n=3)$.

TABLE 3: The calibration curve of liquiritin, deoxyschizandrin, and tanshinone II A under the internal standard method.

\begin{tabular}{|c|c|c|c|c|}
\hline & Range $(\mu \mathrm{g} / \mathrm{mL})$ & Slope $(\text { mean } \pm S D)^{\mathrm{a}}$ & Intercept $(\text { mean } \pm S D)^{\mathrm{a}}$ & $R^{2}$ \\
\hline Liquiritin & $10.00-150.00$ & $(1.5 \pm 0.1) \times 10^{-3}$ & $(4.8 \pm 0.3) \times 10^{-3}$ & 0.9994 \\
\hline Deoxyschizandrin & $15.00-180.00$ & $(1.9 \pm 0.1) \times 10^{-3}$ & $(4.8 \pm 0.2) \times 10^{-3}$ & 0.9984 \\
\hline Tanshinone II A & $5.00-150.00$ & $(1.2 \pm 0.1) \times 10^{-3}$ & $(6.3 \pm 0.2) \times 10^{-3}$ & 0.9992 \\
\hline
\end{tabular}

${ }^{\mathrm{a}}$ Data presented as mean $\pm \mathrm{SD}(n=3)$.

TABLE 4: The precision test results $(n=6)$.

\begin{tabular}{|c|c|c|c|c|c|c|c|c|c|}
\hline \multirow{2}{*}{ Number } & \multicolumn{3}{|c|}{ Liquiritin } & \multicolumn{3}{|c|}{ Deoxyschizandrin } & \multicolumn{3}{|c|}{ Tanshinone II A } \\
\hline & Peak area & Average & RSD (\%) & Peak area & Average & RSD (\%) & Peak area & Average & RSD (\%) \\
\hline 1 & 300.90 & & & 659.60 & & & 270.30 & & \\
\hline 2 & 299.40 & & & 684.10 & & & 276.90 & & \\
\hline 3 & 299.30 & & & 684.80 & & & 268.60 & & \\
\hline 4 & 291.20 & 298.57 & 1.23 & 664.70 & 678.33 & 1.88 & 273.80 & 273.37 & 1.43 \\
\hline 5 & 300.40 & & & 688.70 & & & 278.80 & & \\
\hline 6 & 300.20 & & & 688.10 & & & 271.80 & & \\
\hline
\end{tabular}

TABLE 5: The repeatability test results $(n=6)$.

\begin{tabular}{lccc}
\hline Number & Liquiritin & $\begin{array}{c}\text { Concentration }(\mathrm{mg} / \mathrm{g}) \\
\text { Deoxyschizandrin }\end{array}$ & 0.309 \\
\hline 1 & 0.142 & 0.132 & 0.311 \\
2 & 0.145 & 0.129 & 0.315 \\
3 & 0.141 & 0.126 & 0.310 \\
4 & 0.139 & 0.126 & 0.302 \\
5 & 0.14 & 0.131 & 0.299 \\
6 & 0.138 & 0.127 & 0.308 \\
Average & 0.141 & 0.129 & 1.95 \\
RSD (\%) & 1.76 & 2.00 & \\
\hline
\end{tabular}


TABLE 6: Recovery results of the three biocomponents $(n=6)$.

\begin{tabular}{|c|c|c|c|c|c|c|c|}
\hline Compound & $\begin{array}{l}\text { Sample } \\
(\mathrm{mg})\end{array}$ & $\begin{array}{l}\text { Initial amount } \\
(\mu \mathrm{g})\end{array}$ & $\begin{array}{l}\text { Added amount } \\
(\mu \mathrm{g})\end{array}$ & $\begin{array}{l}\text { Found amount } \\
\qquad(\mu \mathrm{g})\end{array}$ & $\begin{array}{c}\text { Recovery } \\
(\%)\end{array}$ & $\begin{array}{c}\text { Average recovery } \\
(\%)\end{array}$ & $\begin{array}{l}\text { RSD } \\
(\%)\end{array}$ \\
\hline \multirow{6}{*}{ Liquiritin } & 15.98 & 11.37 & \multirow{6}{*}{10.00} & 21.64 & 102.39 & \multirow{6}{*}{100.83} & \multirow{6}{*}{1.52} \\
\hline & 15.95 & 11.35 & & 21.47 & 100.93 & & \\
\hline & 15.92 & 11.33 & & 21.28 & 99.22 & & \\
\hline & 15.71 & 11.18 & & 21.36 & 99.93 & & \\
\hline & 15.65 & 11.13 & & 20.00 & 102.92 & & \\
\hline & 15.42 & 11.37 & & 21.32 & 99.58 & & \\
\hline \multirow{6}{*}{ Deoxyschizandrin } & 15.98 & 6.18 & \multirow{6}{*}{5.00} & 11.42 & 105.28 & \multirow{6}{*}{103.81} & \multirow{6}{*}{1.66} \\
\hline & 15.95 & 6.17 & & 11.36 & 104.08 & & \\
\hline & 15.92 & 6.16 & & 11.34 & 103.68 & & \\
\hline & 15.71 & 6.07 & & 11.40 & 104.88 & & \\
\hline & 15.65 & 6.05 & & 11.38 & 104.48 & & \\
\hline & 15.42 & 5.96 & & 11.18 & 100.48 & & \\
\hline \multirow{6}{*}{ Tanshinone II A } & 15.98 & 29.05 & \multirow{6}{*}{25.00} & 53.50 & 98.05 & \multirow{6}{*}{97.84} & \multirow{6}{*}{1.50} \\
\hline & 15.95 & 28.99 & & 53.08 & 96.35 & & \\
\hline & 15.92 & 28.94 & & 53.06 & 96.27 & & \\
\hline & 15.71 & 28.56 & & 53.36 & 97.47 & & \\
\hline & 15.65 & 28.45 & & 54.00 & 100.03 & & \\
\hline & 15.42 & 28.03 & & 53.71 & 98.87 & & \\
\hline
\end{tabular}

TABLe 7: The stability test results $(n=6)$.

\begin{tabular}{lccc}
\hline Time & & Peak area & Tanshinone II A \\
\hline $0 \mathrm{~h}$ & Liquiritin & Deoxyschizandrin & 927.68 \\
$2 \mathrm{~h}$ & 438.41 & 349.33 & 933.49 \\
$4 \mathrm{~h}$ & 445.9 & 344.85 & 945.12 \\
$8 \mathrm{~h}$ & 440.9 & 345.62 & 930.59 \\
$12 \mathrm{~h}$ & 438.23 & 337.05 & 907.35 \\
$24 \mathrm{~h}$ & 441.56 & 348.9 & 898.63 \\
Mean & 430.28 & 341.93 & 923.81 \\
RSD (\%) & 439.21 & 344.61 & 1.88 \\
\hline
\end{tabular}

${ }^{*}$ Temperature: $25^{\circ} \mathrm{C}$; RH: $40 \%$.

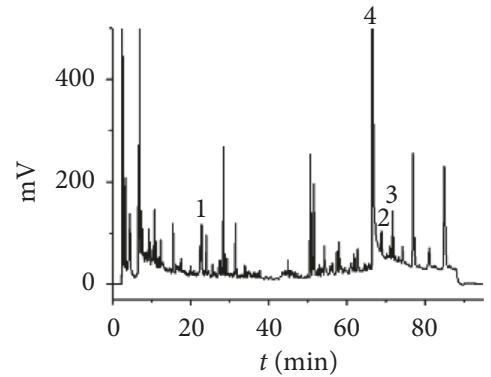

(a)

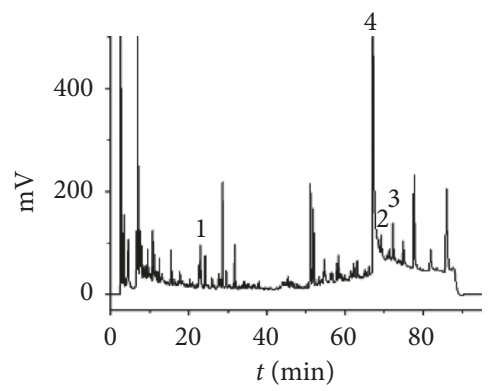

(c)

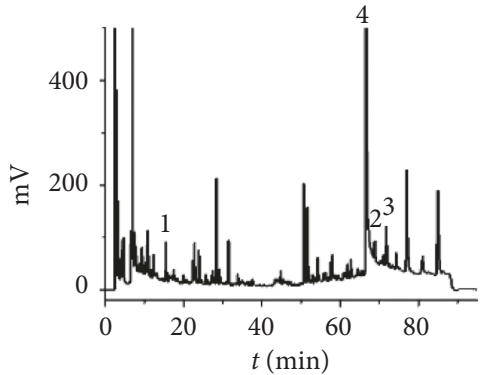

(b)

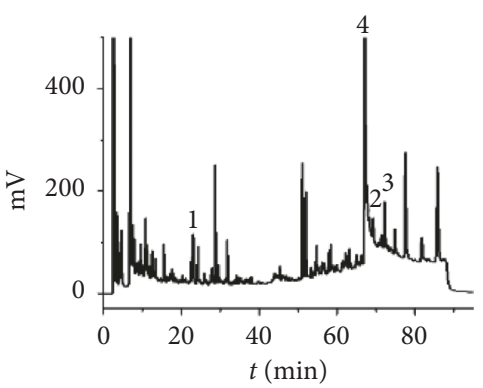

(d)

Figure 2: Continued. 


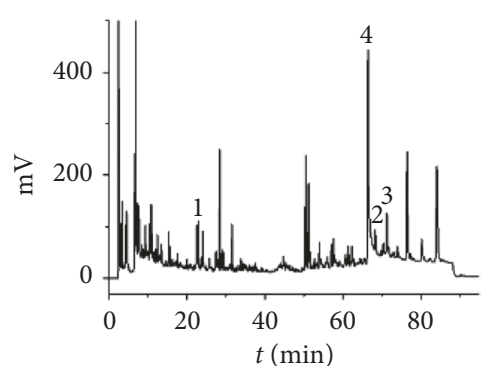

(e)

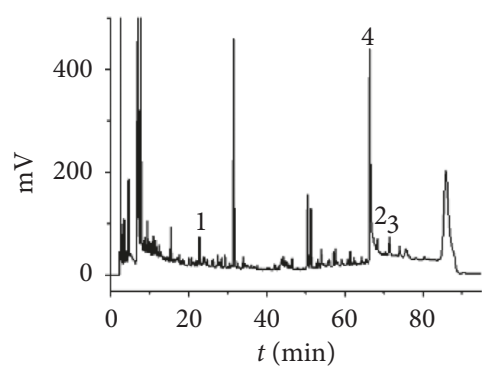

(g)

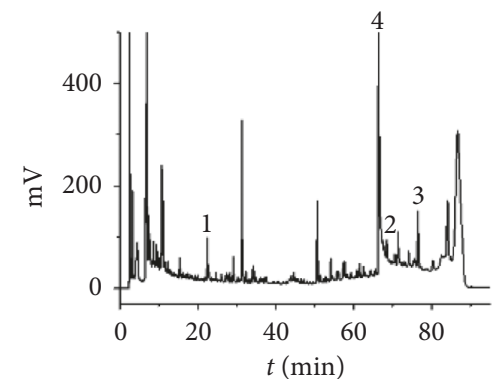

(f)

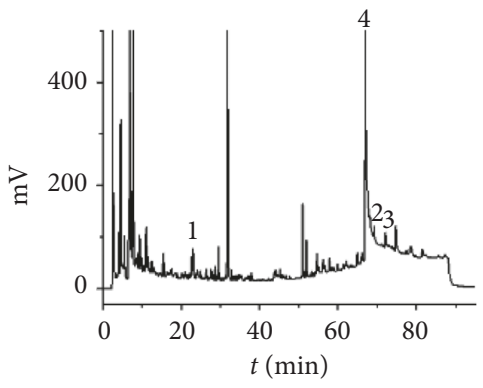

(h)

Figure 2: HPLC chromatograms of eight batches of TWBXP samples: (a) 17052802; (b) 17052803; (c) 17070507; (d) 17091903; (e) 17102402; (f) 17015269; (g) 17055293; (h) 18015225. Peak 1, liquiritin; peak 2, deoxyschizandrin; peak 3, tanshinone II A; peak 4, berberine (IS).

TABle 8: Contents of liquiritin, deoxyschizandrin, and tanshinone II A by the internal standard and external standard method in eight batches of TWBXP samples $(n=6$, mean \pm S.D.).

\begin{tabular}{llccc}
\hline \multirow{2}{*}{ Number } & & \multicolumn{3}{c}{ Content $(\mathrm{mg} / \mathrm{g})$} \\
& Analysis method & Liquiritin & Deoxyschizandrin & Tanshinone II A \\
\hline \multirow{2}{*}{17052802} & External standard & $0.16 \pm 0.01$ & $0.15 \pm 0.01$ & $0.49 \pm 0.01$ \\
& Internal standard & $0.22 \pm 0.01$ & $0.28 \pm 0.01$ & $0.67 \pm 0.01$ \\
17053803 & External standard & $0.14 \pm 0.02$ & $0.12 \pm 0.02$ & $0.42 \pm 0.01$ \\
& Internal standard & $0.19 \pm 0.02$ & $0.22 \pm 0.02$ & $0.58 \pm 0.01$ \\
17070507 & External standard & $0.15 \pm 0.01$ & $0.11 \pm 0.03$ & $0.47 \pm 0.01$ \\
& Internal standard & $0.20 \pm 0.01$ & $0.19 \pm 0.03$ & $0.64 \pm 0.01$ \\
17091903 & External standard & $0.17 \pm 0.02$ & $0.23 \pm 0.02$ & $0.02 \pm 0.02$ \\
17102402 & Internal standard & $0.23 \pm 0.02$ & $0.14 \pm 0.01$ & $0.45 \pm 0.01$ \\
& External standard & $0.16 \pm 0.01$ & $0.21 \pm 0.01$ & $0.12 \pm 0.02$ \\
17015269 & Internal standard & $0.22 \pm 0.01$ & $0.20 \pm 0.02$ & $0.40 \pm 0.02$ \\
17055293 & External standard & $0.15 \pm 0.01$ & $0.11 \pm 0.03$ & $0.56 \pm 0.02$ \\
18015225 & Internal standard & $0.20 \pm 0.01$ & $0.25 \pm 0.03$ & $0.38 \pm 0.03$ \\
& External standard & $0.13 \pm 0.01$ & $0.11 \pm 0.03$ & $0.50 \pm 0.03$ \\
\hline
\end{tabular}

are very low in the TWBXP samples. The contents determined by the internal standard method are higher than that by the external standard method. Therefore, it can be concluded that when the contents of analytes are very low, the internal standard method is preferred to be chosen.

\section{Conclusion}

Two methods for the simultaneous determination of the contents of liquiritin, deoxyschizandrin, and tanshinone II A in TWBXP by HPLC was developed and compared in this study. Both of the proposed method offers the following advantages: the method was simple and convenient in operation without special pretreatment and exhibits excellent precision, repeatability $(\mathrm{RSD}<3.0 \%)$, good linearity $\left(R^{2}>0.9990\right)$, and good recoveries (the recovery value between $95 \%$ and $105 \%$ ). However, when the analytes encountered with low content in the real TCM samples, the internal standard method is superior to the external standard method.

\section{Data Availability}

The data used to support the findings of this study are included within the article. 


\section{Conflicts of Interest}

The authors declare that there are no conflicts of interest.

\section{Acknowledgments}

Prof. Changshan Sun and Prof. Wang Sicen are gratefully acknowledged for the academic guidance. Xi'an Jiaotong University Innovation Improvement Project, Basic Scientific Research Projects in Central Universities (no. PY3A012), and the National Natural Science Foundation of Shaanxi Province (no. 2017JQ8024) are also acknowledged.

\section{References}

[1] H. Liu and J. H. Huang, "The similarities and differences between Suanzaoren decoction and Tianwang Buxin dan in treating deficiency of yin-blood insomnia," Journal of Tianjin University of Traditional Chinese Medicine, vol. 37, pp. 25-27, 2018.

[2] G. T. Gim, H.-M. Kim, J. Kim, J. Kim, W.-W. Whang, and S.-H. Cho, "Antioxidant effect of tianwang buxin pills a traditional Chinese medicine formula: double-blind, randomized controlled trial," The American Journal of Chinese Medicine, vol. 37, no. 2, pp. 227-239, 2009.

[3] R. Ye, Z. Z. Yuan, and C. X. Dai, "Intervention of tianwang buxin decoction combined with dormancy hygiene education for treatment of sub-healthy insomnia patients of yin deficiency fire excess syndrome," Chinese Journal of Integrated Traditional and Western Medicine, vol. 31, p. 618, 2011.

[4] X. M. Li, C. U. Jin, J. P. Zhou et al., "The study of the effect of Tianwang Buxin Wan on model animal behavior and the content of catecholamine neurotransmitter in brain," Pharmacology and Clinics of Chinese Materia Medica, vol. 28, pp. 7-9, 2012.

[5] H. J. Li, Y. Gao, and P. Liu, "Effect of Tianwang Buxin decoction or Tianwang Buxin without radix platycodi decoction on brain neurotransmitter of rats founded hyposomnia model," China Journal of Chinese Materia Medica, vol. 34, p. 217, 2009.

[6] S. J. Liu and Y. L. Zhou, "Ningxin anshen Fang, Ciwujia joint tianwangbuxin treating cardiovascular neurosis randomized controlled study," Journal of Practical Traditional Chinese Internal Medicine, vol. 29, pp. 7-8, 2015.

[7] P. Liu, W. B. Zhou, B. Wu et al., "Effects of Salviae miltiorrhizae and Platycodon grandiflorum in Tianwang Buxin decoction for microcirculation of brain meninx vascullosa in rats," China Journal of Chinese Materia Medica, vol. 32, no. 22, p. 2391, 2007.

[8] G. J. Xie, W. J. Bo, P. P. Huang et al., "Effects of tianwang buxin dan on VIP and AVP expressions of model rats with chronic sleep deprivation," Chinese Archives of Traditional Chinese Medicine, vol. 36, pp. 323-325, 2018.

[9] S.-L. Jia, X.-L. Wu, X.-X. Li et al., "Neuroprotective effects of liquiritin on cognitive deficits induced by soluble amyloid- $\beta 1$ 42oligomers injected into the hippocampus," Journal of Asian Natural Products Research, vol. 18, no. 12, pp. 1186-1199, 2016.

[10] M. F. Zhang, Y. C. Zhao, and Y. Q. Shen, "Research adavances in neuroprotective effects of glycyrrhizae radix et rhizoma and its flavonoids," Anti-Infection Pharmacy, vol. 10, pp. 170-175, 2013.
[11] F. Wei, X. Jiang, H.-Y. Gao, and S.-H. Gao, "Liquiritin induces apoptosis and autophagy in cisplatin (DDP)-resistant gastric cancer cells in vitro and xenograft nude mice in vivo," International Journal of Oncology, vol. 51, no. 5, pp. 1383-1394, 2017.

[12] Z. Zhao, W. Wang, H. Guo, and D. Zhou, "Antidepressantlike effect of liquiritin from Glycyrrhiza uralensis in chronic variable stress induced depression model rats," Behavioural Brain Research, vol. 194, no. 1, pp. 108-113, 2008.

[13] X. D. Huang, J. Ying, Y. C. Wang, and K. Ren, "Anti-aging effects of deoxyschizandrin in D-galactose-induced aging rats," Applied Mechanics and Materials, vol. 423-426, pp. 373-377, 2013.

[14] Y. L. Lu, Q. Q. Hu, L. Chen et al., "Interaction of deoxyschizandrin and schizandrin B with liver uptake transporters OATP1B1 and OATP1B3," Xenobiotica, vol. 49, no. 2, pp. 1-28, 2018.

[15] X. Chen, Y. Y. Zhang, Y. Shao et al., "Inhibitory effect of deoxyschizandrin on growth of brain glioma cells and its mechanism," Journal of Jilin University Medicine Edition, vol. 42, pp. 711-715, 2016.

[16] D. Hu, C. Li, N. Han et al., "Deoxyschizandrin isolated from the fruits of schisandra chinensis ameliorates a $\beta 1-42$-induced memory impairment in mice," Planta Medica, vol. 78, no. 12, pp. 1332-1336, 2012.

[17] P. Yang, Y. H. Jia, J. Li et al., "Anti-oxidation of tanshinone II A and prohibitin on cardiomyocytes," Chinese Herbal Medicines, vol. 2, pp. 204-210, 2010.

[18] C. Guo, Y. Yin, J. Duan et al., "Neuroprotective effect and underlying mechanism of sodium danshensu [3-(3,4-dihydroxyphenyl) lactic acid from radix and rhizoma salviae miltiorrhizae $=$ danshen] against cerebral ischemia and reperfusion injury in rats," Phytomedicine, vol. 22, no. 2, pp. 283-289, 2015.

[19] S. F. Nabavi, G. C. Tenore, M. Daglia et al., "The cellular protective effects of rosmarinic acid: from bench to bedside," Current Neurovascular Research, vol. 12, pp. 98-105, 2014.

[20] J. H. Park, O. K. Park, B. Yan et al., "Neuroprotection via maintenance or increase of antioxidants and neurotrophic factors in ischemic gerbil hippocampus treated with tanshinone I," Chinese Medical Journal, vol. 127, pp. 3396-3405, 2014.

[21] J. Li, F. Wang, J. Zhou et al., "Effects of tanshinone II A on the expressions of p53, pp53 and apoptosis in the rats with Alzheimer's disease," Journal of Central South University, vol. 40, pp. 1210-1216, 2015.

[22] C. Liu, Y. Wu, S. Zha et al., “Treatment effects of tanshinone II A against intracerebroventricular streptozotocin induced memory deficits in mice," Brain Research, vol. 1631, pp. 137-146, 2016.

[23] F. Li, G. Han, and K. Wu, “Tanshinone II A alleviates the AD phenotypes in APP and PS1 transgenic mice," BioMed Research International, vol. 2016, Article ID 7631801, 8 pages, 2016.

[24] P. Jiang, C. Li, Z. Xiang, and B. Jiao, “Tanshinone II A reduces the risk of Alzheimer's disease by inhibiting iNOS, MMP-2 and NF- $\kappa$ Bp 65 transcription and translation in the temporal lobes of rat models of Alzheimer's disease," Molecular Medicine Reports, vol. 10, no. 2, pp. 689-694, 2014.

[25] Y. Yin, L. Huang, Y. Liu et al., "Effect of tanshinone on the levels of nitric oxide synthase and acetylcholinesterase in the brain of alzheimer's disease rat model," Clinical and Investigative Medicine, vol. 31, no. 5, pp. 248-257, 2008. 
[26] X. L. Sun, Q. Z. Zhu, and F. He, "Simultaneous determination of six lignans in tianwang buxin pill by RRLC," Chinese Pharmaceutical Affairs, vol. 30, pp. 1112-1116, 2016.

[27] Y. Yan, L. Shi, N. Shi et al., "Simultaneous determination of four components of tianwangbuxin tables by UPLC-Q-TOFMS," Pharmaceutical Journal of Chinese People's Liberation Army, vol. 34, pp. 55-57, 2018. 

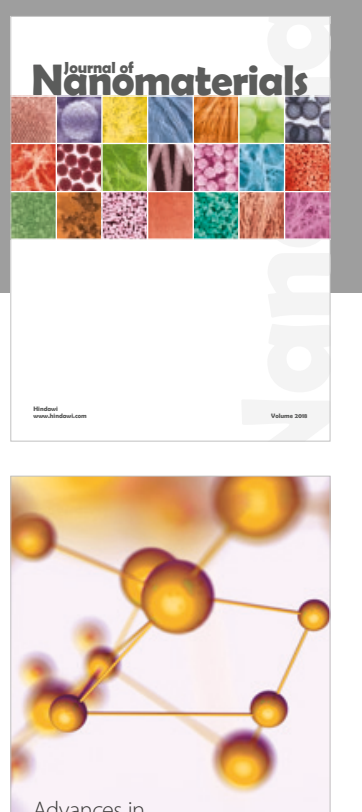

Physical Chemistry
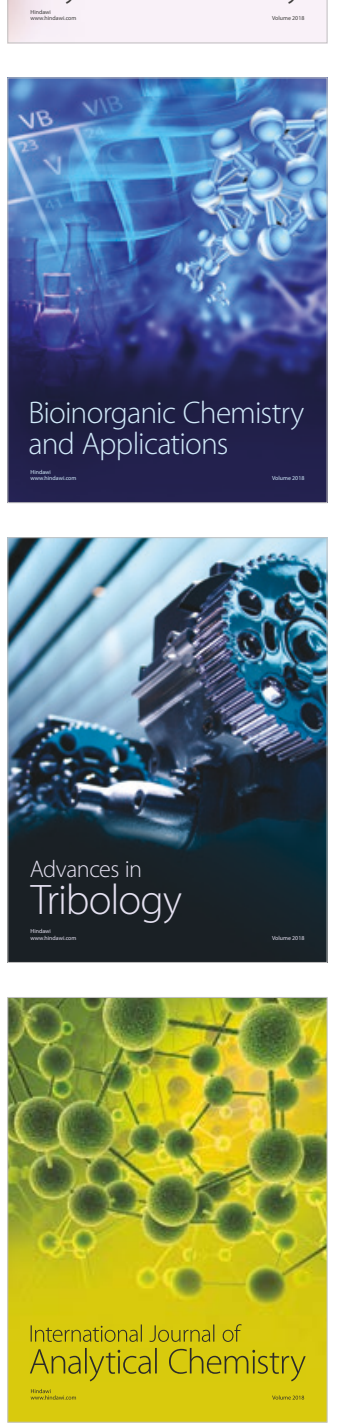

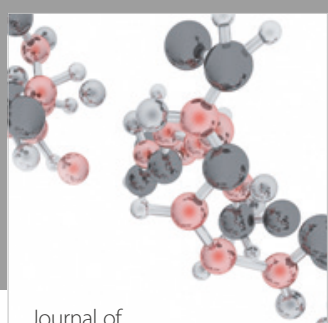

Analytical Methods

in Chemistry

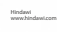

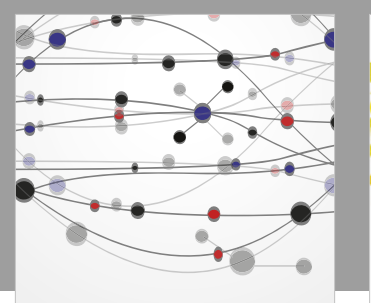

The Scientific World Journal

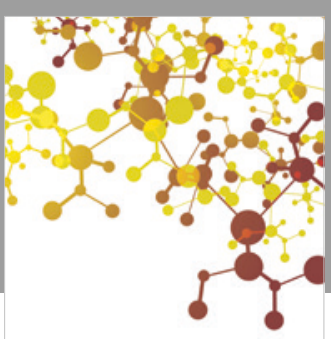

Journal of

Applied Chemistry
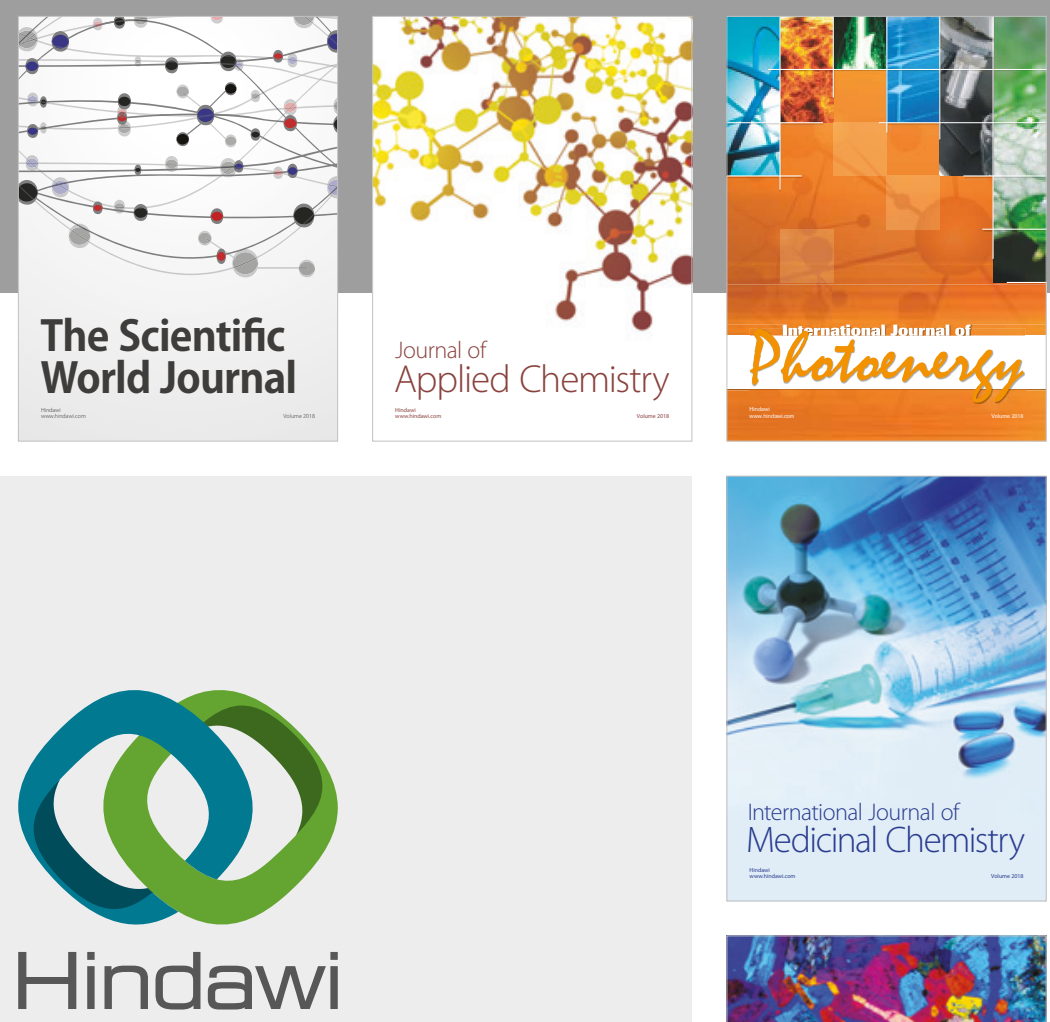

Submit your manuscripts at

www.hindawi.com
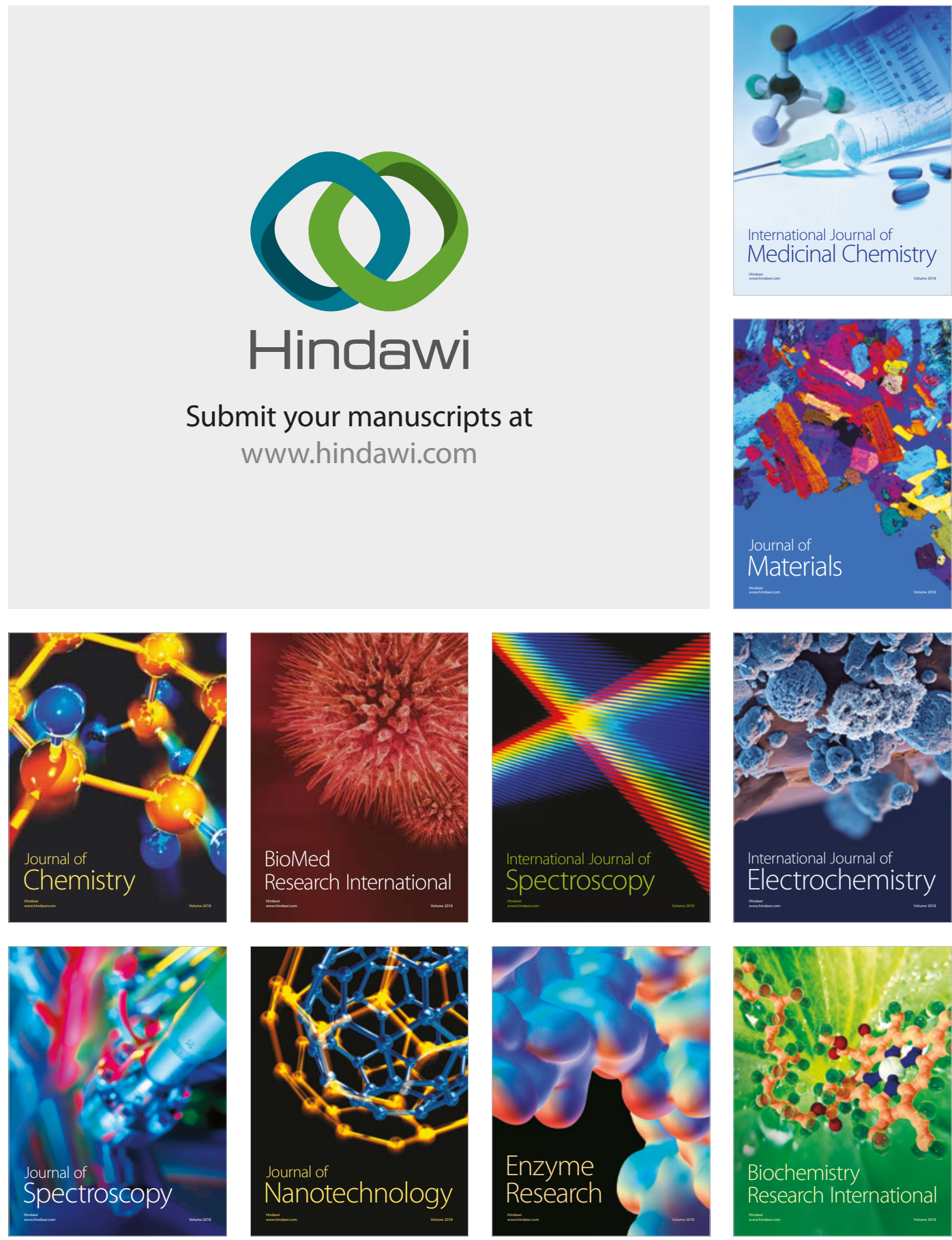
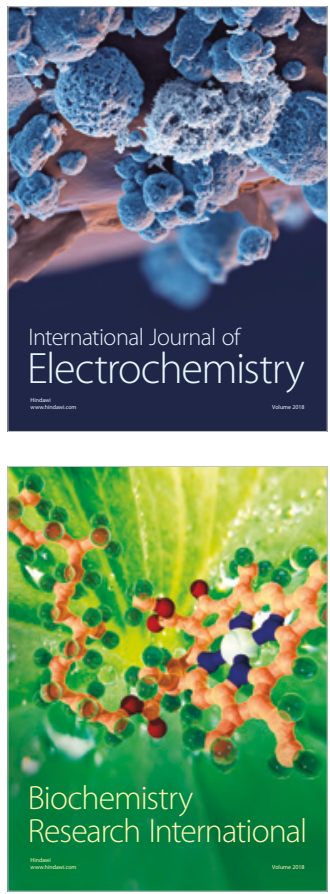